\title{
Adverse effects associated with the short-term treatment of panic disorder with imipramine, alprazolam or placebo
}

\author{
Giovanni B. Cassano ${ }^{\mathrm{a},}{ }^{*}$, Cristina Toni $^{\mathrm{a}}$, Alberto Petracca ${ }^{\mathrm{a}}$, Joseph Deltito ${ }^{\mathrm{b}}$, Otto Benkert ${ }^{\mathrm{c}}$, \\ George Curtis ${ }^{\mathrm{d}}$, H. Hippius ${ }^{\mathrm{e}}$, Wolfgang Maier ${ }^{\mathrm{c}}$, David Shera ${ }^{\mathrm{f}}$, Gerald Klerman ${ }^{\mathrm{b},}$

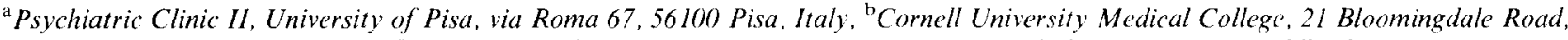 \\ White Plains, NY 10605, USA, "Department of Psychiatry, University of Mainz, Postfach 3960, Untere Zahlbacher Street 8, 6500 \\ Mainz, Germany, ${ }^{\mathrm{d}}$ University of Michigan Medical Center, Department of Psychiatry, Med. Inn Building 444, 1500 East Medical Center \\ Drive, Ann Arbor, MI 48109-0804, USA, ${ }^{\circ}$ Psychiatric Clinic, University of Munich, Nussbaumstrasse 7, 8000 Munich 2, Germany, ${ }^{\mathrm{f}}$ New \\ England Biomedical Research Foundation, Cambridge, MA 02238-2732, USA
}

(Received 30 March 1993)

(Revision received 5 October 1993)

(Accepted 5 October 1993)

Key words: Panic disorder; Imipramine; Alprazolam; Side effects; Adverse reactions; Compliance

\section{Summary}

Side effects play a significant role in the selection of drugs to be used in panic disorder/agoraphobia whose polyphobic symptomatology often includes a suspiciousness about taking drugs and a fear of undesired side effects which may lead to the refusal of treatment.

The safety, side effects and patients' acceptance of alprazolam and imipramine versus placebo were evaluated in 1168 subjects with panic disorder/agoraphobia who had been enrolled in the second phase of the Upjohn World Wide Panic Study. Side effects that worsened over baseline to a greater extent with alprazolam than with imipramine and placebo were sedation, fatigue/weakness, memory problems, ataxia and slurred speech. In the imipramine group blurred vision, tachycardia/palpitations, insomnia, sleep disturbance, excitement/nervousness, malaise, dizziness/faintness, headache, nausea/vomiting and decrease in appetite were worse than in the other groups. In the placebo group the anxious symptoms were most prominent. The highest level of compliance was shown in the alprazolam-treated group and the lowest in the placebo-treated group. Strong predictors of side effects were not observed.

If a side effect profile is known, it will be easier for a clinician to choose the right drug and the appropriate management by taking into account compliance, safety and efficacy in each patient under treatment. Further information about side effects in long-term maintenance treatment would be of great clinical pertinence in ensuring safety and enhancing patients' quality of life.

\section{Introduction}

Currently available psychopharmachological

\footnotetext{
"Corresponding author.

† Professor Klerman died in July 1992.
}

compounds enable us to treat a large number of patients who, in the past, would have been classified as non-responders to any kind of psychotherapeutic or somatic treatment.

Tricyclic antidepressants (TCAs) have been used in the treatment of panic disorder/agoraphobia since Donald Klein (1962) first noted their clinical 
efficacy. His further studies in this area led to these diseases being located within the framework of anxiety disorders. Several studies confirmed the efficacy of imipramine (Sheehan et al., 1980; Mavissakalian et al., 1986; Schweizer et al., 1993) and of other drugs such as phenelzine (Sheehan et al., 1984), clomipramine (Gloger et al., 1981; Grunhaus et al., 1984; Cassano et al., 1988), alprazolam (Chouinard et al., 1983; Sheehan et al., 1984; Charney et al., 1986), clonazepam (Pollack, 1990; Tesar, 1990) and the selective serotonin reuptake inhibitors (SSRI) fluvoxamine and fluoxetine (Gorman et al., 1987; Black et al., 1993).

More precise information concerning both longand short-term safety will greatly help the clinician in choosing between new drugs of equal or nearly equal efficacy.

Side effects play a significant role in the selection of drugs to be used in anxiety disorders whose polyphobic symptomatology often includes a suspiciousness about taking drugs and a fear of undesired side effects which may lead to the refusal or unduly early interruption of treatment.

In 1982 the Upjohn Company proposed a large multicenter study in the field of anxiety disorders: the Cross-National Collaborative Panic Study, a two-phase controlled clinical trial (Klerman et al., 1986). The results of the first phase, comparing alprazolam against placebo, were published in 1988 by Ballenger and associates. Noyes and associates and Pecknold and associates. Patient acceptance, side effects, and safety were described in detail by Noyes et al. (1988). Patient acceptance was higher for alprazolam than placebo $(84 \%$ vs. $50 \%)$. The treatment-related side effects which were most frequently recorded in the alprazolam group included sedation, fatigue, ataxia, slurred speech, and amnesia. Ten cases of serious adverse drug reactions (acutc intoxication, hepatitis, mania, amnesia, aggressive behavior, and depression) occurred among the 263 patients taking alprazolam in the first phase.

The second phase studied the relative safety and efficacy of alprazolam (ALP) versus imipramine (IMI) and placebo (PBO) in controlling panic attacks and attendant avoidance behavior in outpatients with panic disorder which was complicated in some cases by various levels of avoidance behavior.

Of all the studies carried out so far, the present one has certainly involved the greatest participation of investigators and the highest number of patients (1168) receiving psychoactive compound treatment for an anxiety disorder (panic disorder). It provided a unique opportunity for evaluating the side effect profile of ALP and IMI.

The following report focuses on data concerning the safety, side effects, adverse reactions and patients' acceptance of ALP, IMI and PBO.

\section{Subjects and method}

Twelve centers in Europe and North and South America took part in the second phase of the Cross-National Collaborative Panic Study, which compared ALP, IMI and PBO in the treatment of panic disorder with various accompanying levels of agoraphobia (Cross-National Collaborative Panic Study Second Phase Investigators, 1992). 1168 subjects with uncomplicated panic disorder, panic disorder with limited phobic avoidance, or panic disorder with extensive phobic avoidance (agoraphobia), according to DSM-III criteria, were enrolled. Patients had to participate voluntarily, be able to take oral medication, be of non-childbearing potential or use contraceptive precautions and be between 18 and 65 years of age. Patients with any of the following conditions were excluded: acute suicidal ideation, pregnant or lactating, a history of epilepsy, acute or unstable medical problems, comorbidity with other psychiatric disorders. Patients taking drugs acting on the central nervous system, including benzodiazepines, or undergoing any psychotherapy were excluded from the study.

The sample was homogeneous with respect to all demographic and clinical variables (Cross- $\mathrm{Na}$ tional Collaborative Panic Study Second Phase Investigators, 1992).

Before randomization to drug group, patients were withdrawn from all drugs acting on the central nervous system, in a wash-out period of 1 or 2 weeks. Then, a random assignment was carried out for double-blind treatment with ALP, IMI or PBO over an 8-week period: 386 subjects were allocated to the ALP group, 391 to the IMI group, and 391 to the PBO group. 1-mg capsules of ALP or identical capsules containing $25 \mathrm{mg}$ of IMI or PBO were administered according to a gradually increasing dosage schedule. Whenever possible, the patients were brought to the most effective dose by the end of the third week. In the case of side effects. the dosage could be reduced until the medication was better tolerated by the patient, after which 
dosage increases were resumed until at least six capsules per day were being taken - the maximum dosage allowed being 10 capsules per day. If patients were unable to tolerate six capsules after repeated attempts to maintain the dose, the treating physician had the option of maintaining the patient on the maximally tolerated dose.

Throughout the trial, the number of ALP capsules was lower than that of the other drugs. At the endpoint, the values for PBO were higher than those for ALP and for IMI (5.5 capsules for ALP, 6.1 capsules for IMI, 6.4 capsules for PBO).

Subject response to medication and the development of side effects were evaluated at weeks 1,2, $3,4,6$ and 8 .

Information on treatment emergent symptoms was collected with the SAFTEE-UP, a brief structured interview derived from the SAFTEE rating system developed by Levine et al. (1986). At baseline and at the subsequent visits all the symptoms observed by means of the SAFTEEUP interview were recorded on a 42 -item checklist, the 'Symptoms and Side Effects Checklist'. Each symptom was rated as 'absent', 'mild', 'moderate' or 'severe'.

Whenever a serious adverse effect occurred, it was entered in the 'Medical Events' form. This was used to report all serious or unexpected medical events that occurred during the study, independently of any assessment of how the events might be related to the study medication.

For patients who failed to complete the study, the reasons for termination were recorded and symptoms indicative of adverse drug effects were assessed during their last visit.

Side effects experienced by subjects receiving ALP, IMI or PBO were compared with respect to change from baseline for each week of the study.

Moreover, given all the data collected on side effects, it was reasonable to suppose that some of the analyses might offer information directly pertinent to the clinical setting on whether there were predictors (e.g., age, sex, subtype of disorder, prior benzodiazepine use and site of the study) of which types of patients would suffered most side effects and from which agents.

Regressions and chi square tests were employed for a comparative analysis of the side effects recorded in the treatment groups and to obtain information about predictors.

\section{Results}

\section{Treatment-related symptoms}

Side effects worsened versus baseline. Most symptoms worsened, compared to baseline, more markedly during the first week of the study than at the middle (week 4) and at the end (week 8) of the trial. 'Sedation', 'fatigue/weakness', 'memory problems', 'ataxia' and 'slurred speech' showed an increase versus baseline severity more in patients taking ALP than in those taking IMI or PBO until the very end of the study (Table 1).

'Irritability', despite a greater similarity in the degree of worsening found in the three groups during the first week, confirmed the general trend of a higher percentage in the ALP group both after the fourth week and the eighth.

'Increase in appetite' followed the global trend of a greater worsening during the first week than during the fourth or eighth, but, except for the first week, it was not more marked in the ALP group than in the other two; the IMI group showed an increase in this side effect as the trial progressed. Similarly, 'weight gain' was greater in the ALP group during the first week, but by the fourth was similar to that found in the other two groups.

Anticholinergic side effects and symptoms of adrenergic overactivity worsened more in the IMI group than in the ALP group during the first week, but showed a marked reduction from the fourth week onwards (Table 1). Tolerance did not develop to some anticholinergic side effects, such as 'dry mouth', 'constipation', 'difficulty urinating' and 'excessive sweating'.

In the PBO group, baseline symptoms showed a milder aggravation than those of other treatment groups; the only exception was the anxious symptoms (dizziness/faintness, hyperventilation, chest pain, and stomach/abdominal discomfort) (Table 1).

Side effects associated with discontinuation of treatment prior to 8 weeks. $17.4 \%$ of subjects in the ALP group, $30.2 \%$ in the IMI group, and $43.7 \%$ in the PBO group did not complete the study. Side effects were only infrequently the cause of failure to complete the protocol: only $3.4 \%$ subjects taking ALP, 5.9\% taking IMI and 3.1\% taking PBO interrupted the study because of side effects. Other reasons for treatment interruption within the 8-week protocol are reported in Table 2.

The anticholinergic side effects, usually reported during tricyclic antidepressant treatment, showed 
TABLE 1

PERCENTAGE OF SUBJECTS REPORTING AN INCREASE IN SIDE EFFECTS OVER BASELINE

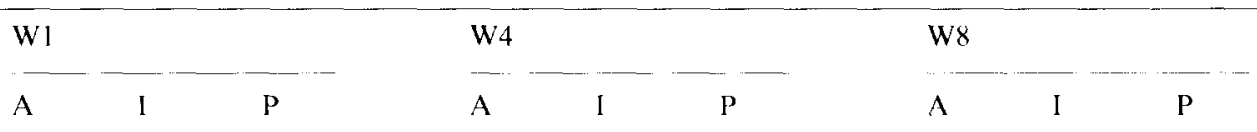

Side effects whose aggravation was more severe with alprazolam than imipramine

Sedation

Fatigue/weakness

Irritability

Memory problems

Ataxia

Slurred speech

Increased appetite

Weight gain

\begin{tabular}{lrr}
$\frac{58}{20}$ & $\frac{31}{19}$ & $\frac{21}{16}$ \\
12 & 13 & 11 \\
16 & 13 & 11 \\
$\frac{16}{7}$ & $\frac{6}{4}$ & $\frac{8}{4}$ \\
$\frac{21}{11}$ & $\frac{4}{2}$ & $\frac{12}{2}$ \\
\hline
\end{tabular}

$\begin{array}{rrr}\frac{44}{18} & \frac{20}{13} & \frac{16}{12} \\ \frac{14}{15} & \frac{7}{9} & \frac{8}{9} \\ \frac{9}{7} & \frac{2}{1} & \frac{4}{3} \\ \frac{7}{13} & 1 \frac{3}{5} & 1 \frac{6}{12} \\ 12 & 12 & 6\end{array}$

$\begin{array}{rrr}\frac{29}{13} & \frac{10}{7} & \frac{11}{9} \\ 11 & 7 & 8 \\ \frac{14}{8} & \frac{7}{1} & \frac{5}{2} \\ \frac{1}{4} & \frac{1}{1} & \frac{1}{1} \\ 8 & 14 & 7 \\ 8 & 14 & 11\end{array}$

Side effects whose aggravation was greater with imipramine than alprazolam

Insomnia

Other sleep disturbance

Nervousness

Malaise

Dizziness/faintness

Headache

Blurred vision

Dry mouth

Tremor

Tachycardia

Nausea/vomiting

Constipation

Difficulty urinating

Excessive sweating

Decreased appetite

\begin{tabular}{|c|c|}
\hline 3 & 22 \\
\hline$\underline{4}$ & $\overline{14}$ \\
\hline$\underline{6}$ & $\overline{16}$ \\
\hline$\underline{6}$ & 12 \\
\hline $1 \frac{5}{5}$ & 22 \\
\hline 13 & 21 \\
\hline$\underline{9}$ & 16 \\
\hline $1 \overline{6}$ & $\underline{52}$ \\
\hline 8 & $\overline{14}$ \\
\hline$\underline{6}$ & $\underline{18}$ \\
\hline $\bar{z}$ & 17 \\
\hline $1 \overline{2}$ & 18 \\
\hline$\underline{1}$ & $\underline{6}$ \\
\hline$\overline{9}$ & $2 \overline{7}$ \\
\hline 0 & $\overline{17}$ \\
\hline
\end{tabular}

$\begin{array}{rrr}\frac{5}{5} & \frac{11}{10} & \frac{12}{8} \\ 9 & 8 & 10 \\ 6 & 6 & 8 \\ 10 & 11 & 14 \\ 15 & 13 & 13 \\ \frac{5}{7} & \frac{15}{51} & \frac{10}{15} \\ \frac{5}{3} & \frac{51}{7} & \frac{9}{8} \\ \frac{13}{3} & \frac{31}{9} & \frac{6}{3} \\ \frac{3}{6} & \frac{2}{9} & \frac{11}{8}\end{array}$

$\begin{array}{rrr}4 & 7 & 8 \\ 5 & 6 & 6 \\ \frac{7}{5} & \frac{4}{4} & \frac{12}{5} \\ 6 & 7 & 9 \\ 11 & 7 & 11 \\ 5 & 5 & 6 \\ \frac{11}{6} & \frac{40}{10} & \frac{7}{5} \\ \frac{6}{3} & \frac{10}{4} & \frac{5}{6} \\ \frac{6}{12} & \frac{23}{9} & \frac{5}{1} \\ \frac{4}{4} & \frac{19}{5} & \frac{1}{7} \\ \frac{1}{7} & \frac{7}{6} & \end{array}$

Side effects whose aggravation was greater with placebo than other groups

Dizziness/faintness

Hyperventilation

Chest pain

Abdominal discomfort

$\begin{array}{ccc}\text { as greater with place ho than other gro } \\ 15 & 22 & 18 \\ 7 & 8 & 12 \\ \frac{7}{9} & \frac{11}{12} & \frac{13}{16}\end{array}$

\begin{tabular}{rrr}
10 & 11 & 14 \\
5 & 5 & 7 \\
5 & 6 & 10 \\
$\underline{8}$ & $\underline{8}$ & $\underline{16}$ \\
\hline
\end{tabular}

\begin{tabular}{rrr}
6 & 7 & 9 \\
5 & 4 & 8 \\
8 & 4 & 7 \\
8 & 8 & 10 \\
\hline
\end{tabular}

For underlined values, statistically significant differences were observed using the chi square test.

$\mathrm{A}=$ alprazolam; $\mathrm{I}=$ imipramine $\mathrm{P}=$ placebo $\mathrm{W}=$ week

no difference between the three treatment groups in causing trial interruptions. Conversely, a greater frequency of trial interruption was attributed to anxious symptoms in the PBO group, mostly during the first 4 weeks of the study. 'Tachycardia' $(\mathrm{ALP}=0 \%, \mathrm{IMI}=1.3 \%, \mathrm{PBO}=2.3 \%)$, "chest pain' $(A L P=0 \%, I M I=0 \%, P B O=1 \%)$, 'insomnia' $\quad(\mathrm{ALP}=0.2 \%, \quad \mathrm{IMI}=0.8 \%, \quad \mathrm{PBO}=2.3 \%)$, 'excitement' $(\mathrm{ALP}=0.8 \%, \quad \mathrm{IMI}=1.3 \%$, $\mathrm{PBO}=2.5 \%)$ and 'headache' (ALP $=0.5 \%$, $\mathrm{IMI}=0 \%, \mathrm{PBO}=1.5 \%$ ) were significantly prevalent in the PBO group.

Side effects usually associated with benzodiazepine administration, like 'sedation' (ALP $=2.3 \%$, $\mathrm{IMI}=0.2 \%, \mathrm{PBO}=0.2 \%)$, 'ataxia' (ALP $=1.0 \%$, $\mathrm{IMI}=0 \%, \mathrm{PBO}=0 \%$ ) and 'memory problems'
$(\mathrm{ALP}=1.0 \%, \quad \mathrm{IMI}=0 \%, \mathrm{PBO}=0 \%)$ caused several interruptions mostly in the ALP group.

In all cases, a combination of symptoms mostly caused the treatment interruption.

TABLE 2

REASONS FOR TERMINATION

\begin{tabular}{llrr}
\hline & $\mathrm{A}(\%)$ & $\mathrm{I}(\%)$ & $\mathrm{P}(\%)$ \\
\hline Side effects & 3.4 & 5.9 & 3.1 \\
Ineffectiveness & 3.1 & 2.6 & 12.8 \\
Refused treatment & 3.9 & 14.1 & 16.1 \\
Other & 7.0 & 7.7 & 11.8 \\
\hline
\end{tabular}

$\mathbf{A}=$ alprazolam; $\mathbf{I}=$ imipramine; $\mathbf{P}=$ placebo. 
TABLE 3

ADVERSE REACTIONS

\begin{tabular}{|c|c|c|c|}
\hline & $A(N)$ & $I(N)$ & $P(N)$ \\
\hline Confusion & 3 & 1 & \\
\hline Abnormal liver tests & 2 & 4 & \\
\hline Breast cancer & & & 2 \\
\hline Melena & 1 & & \\
\hline Depression & 4 & 1 & 3 \\
\hline Mental stimulation & & 1 & \\
\hline Disinhibition & 1 & & \\
\hline Aggressive behavior & & 2 & \\
\hline Hallucinations & 1 & 2 & 1 \\
\hline Seizure & 1 & & \\
\hline
\end{tabular}

$\mathrm{A}=$ alprazolam $\mathbf{I}=$ imipramine $\mathrm{P}=$ placebo $\mathrm{N}=$ number of patients.

\section{Adverse reactions}

Only few adverse reactions were reported (Table 3).

There were no episodes of acute intoxication, cardiac or renal illness or amnesia.

A severe seizure was observed in one patient taking $6 \mathrm{mg}$ of ALP. The etiology of the seizure was unknown, but it was not related to missed doses or unauthorized reductions of dosage.

Major depression, according to DSM-III criteria, occurred in eight patients (four patients in the ALP group, three patients in the PBO group and one patient in the IMI group). There were no cases of mania.

\section{Side effect predictors}

We did not observe any relevant side effect predictors. Only occasionally we have observed limited effects due to age, sex, panic type, prior benzodiazepine use, site of the study; none of these factors overshadowed the main effect of medication.

\section{Comment and concluding remarks}

In many ways panic disorder/agoraphobia is a unique condition in which differences between the side effect spectra of different drugs are of great clinical pertinence not only to safety, but also to a patient's quality of life in short- and long-term maintenance treatments. The sensibility of these patients is such that they are likely to become drugphobic and drug-avoidant - a situation where a high rate of attrition occurs if there are unexpected side effects or any nonspecific symptoms that the patient may attribute to the administered compound. If a side effect profile is well known to the clinician, it will be easier for him or her to choose both the right drug and the appropriate management by taking into account compliance, safety and efficacy in each patient under treatment.

The rigorous methodology of this study and the large sample of patients selected in different countries using an identical protocol allow us to analyze the prevalence and severity of the spectrum of side effects during the treatment of panic disorder/agoraphobia with ALP and IMI.

Our data mostly confirm previous investigations in which ALP, IMI and PBO were studied in the treatment of depression or panic disorder (Fabre et al., 1980; Feighner et al., 1983; Mendels et al., 1986; Rizley et al., 1986). In these studies, patients on ALP reported fewer side effects than those on IMI. Anticholinergic effects and overstimulation have been largely reported as the most frequent side effects during IMI treatment (Noyes et al., 1989), while a side effect profile similar to that seen with other benzodiazepines (Aden et al., 1980; Cohn 1981; Kolin et al., 1981) has been described with ALP treatment. Surprisingly enough, in our study the 'anticholinergic side effects' reported in the IMI group failed to reach levels as high as those recorded in previous observations on depressives (Fabre et al., 1980; Feighner et al., 1983; Mendels et al., 1986).

The severity of side effects decreased over the 8 weeks of treatment in all three groups; in most cases both their number and severity had dropped after 3 or 4 weeks. This observation is in agreement with the findings of other authors (Mendels et al., 1986; Noyes et al., 1988). This is probably at least partly due to patients dropping out in the third week because of side effects, so that values after week 4 are probably biased towards lower side effects, especially in the two groups whose completion rate was lowest.

The dropout rate in the ALP group was significantly lower than that in the IMI and PBO groups. This finding must be considered in the context of the high doses of ALP which were employed. The low dropout rate in the ALP group suggests a high level of patient acceptance of ALP treatment. A high percentage of the patients who took ALP but failed to complete the study showed symptoms related to benzodiazepine administration, and it is likely that an initially lower dose, or a more gradual dosage increase, would have reduced 
the incidence of these side effects.

In the IMI group, few patients stopped treatment because of side effects, mostly anticholinergic and overstimulation symptoms. However, anticholinergic side effects did not cause statistically significant treatment interruption in any of the three groups.

Few adverse reactions to ALP and IMI were observed in this study. Apart from a case of seizure in the ALP group, there were no episodes either of serious somatic illness, or of laboratory abnormalities that were strictly related to the drug being administered and also responsible for treatment interruption.

Depression has been observed with the use of benzodiazepines and has been reported in a minority of patients with panic disorder receiving high doses of ALP (Greenblatt et al., 1974; Lydiard et al., 1987). One case of depression was reported in phase I of this study, too (Noyes et al., 1988). These few findings, however, do not allow us to assert that there is definitely a relationship between depression and benzodiazepine treatment. Unlike phase I, phase II of this study included no cases of mania, in either the IMI or the ALP group, but only aspecific signs of mental stimulation. In our large sample, only one patient treated with ALP was reported to have developed aspecific symptoms of mental disinhibition; neither aggressiveness nor a clear-cut manic or hypomanic state was noticed in any of the ALP group of patients. The results of the present study failed to confirm previous observations on a possible induction of mania by ALP (Arana et al., 1985; Strahan et al., 1985). It should, however, be recalled that the selection criteria for our sample excluded patients with a history of bipolar disorder.

Regarding possible side effect predictors, we have observed occasionally limited effects due to age, sex, panic type, prior benzodiazepine use, site of the study; none of these factors overshadowed the main effect of medication.

Given there were so many models tested, it is still very likely that differences would be significant just by chance. Moreover, the study was designed to find the impact of the treatments, not the effects of the different factors. Therefore the number of patients is really only sufficient for the former main purpose, and confirmation of other effects would probably require a study on a larger scale.

The side effect profile of ALP and IMI, studied by comparison with $\mathrm{PBO}$, is to be completed in a subsequent publication by reporting the data from an extension period lasting an extra 6 months during which these drugs were administered to a limited number of patients under similar experimental conditions.

Previous observations with the use of IMI for extended periods showed that anticholinergic effects and overstimulation were frequent during the early phase of treatment and reduced patients' acceptance of the drug; after the initial period, weight gain was the most frequent side effect leading to discontinuation of the treatment (Noyes et al., 1989). This observation has been confirmed by preliminary analyses on side effects during the extension period of treatment of the Upjohn Study (Curtis et al., 1993). Regarding ALP, information about its effectiveness and safety over the long term is still insufficient. Preliminary observations focused on tolerance and withdrawal problems, but further studies are needed (Burrows, 1990; Davidson, 1990; Pollack, 1990). Moreover, preliminary analyses on the extension Upjohn Study data showed that the incidence of side effects decreased or remained unchanged with long-term therapy with ALP; those most frequently reported were weight loss, change in libido, headache and depressed mood (Burrows, 1990).

The differences in the types and rates of side effects appearing in the early and late phases of treatment with the various compounds under study should indicate that a well-targeted patient management plan should be formulated not only for each drug, but also for each treatment period.

Even if no definitive information on the longterm therapy of panic disorder is available yet, this study has clearly shown that in the early phase of treatment IMI and ALP are both well tolerated, causing small percentages of side effects and adverse reactions.

\section{Acknowledgement}

Supported by grants from the Upjohn Company, Kalamazoo, Michigan.

\section{References}

Aden, G.C. and Thein, S.G. (1980) Alprazolam compared to diazepam and placebo in the treatment of anxiety. J. Clin. Psychiatry 41, 245- 248.

Arana, G.W., Pearlman, C. and Shader, R.I. (1985) Alprazolam-induced mania: two clinical cases. Am. J. Psychiatry $142,368-369$. 
Ballenger, J.C., Burrows, G.D., Dupont, R.L. Jr., Lesser, I.M., Noyes, R., Pecknold, J.C., Rifkin, A. and Swinson, R.P. (1988) Alprazolam in panic disorder and agoraphobia: Results from a multicenter trial. Efficacy in short-term treatment. Arch. Gen. Psychiatry 45, 413-422.

Black, D.W., Wesner, R., Bowers, W. and Gabel, J. (1993) A comparison of fluvoxamine, cognitive therapy and placebo in the treatment of panic disorder. Arch. Gen. Psychiatry $50,44-50$.

Burrows, G.D. (1990) Managing long-term therapy for panic disorder. J. Clin. Psychiatry 51, 9-12.

Cassano, G.B., Petracca, A., Perugi, G., Nisita, C., Musetti, L., Mengali, F. and McNair, D.M. (1988) Clomipramine for panic disorder: 1. The first 10 weeks of a long term comparison with imipramine. J. Affect. Disord. 14, 123 127.

Charney, D.S., Woods, S.W., Goodman, W.K., Rifkin, B., Kinch, M., Aiken, B., Quadrino, L.M. and Heninger, G.R. (1986) Drug treatment of panic disorder: the comparative efficacy of imipramine, alprazolam and trazodone. J. Clin. Psychiatry 47, 580-586.

Chouinard, G., Annable, L., Fontaine, R. and Solyom, L. (1983) Alprazolam in the treatment of generalized anxiety and panic disorder: a double blind placebo controlled study. Psychopharmacology 77, 229-223.

Cohn, J.B. (1981) Multicenter double blind efficacy and safety study comparing alprazolam, diazepam, and placebo in clinically anxious patients. J. Clin. Psychiatry 42, 347-351.

Cross National Collaborative Panic Study Second Phase Investigators (1992) Drug treatment of panic disorder. $\mathrm{Br}$. J. Psychiatry 160, 191-202.

Curtis, G., Massana, J., Udina, C., Ayuso, J.L., Cassano, G.B. and Perugi, G. (1993) Maintenance drug therapy of panic disorder. J. Psychiatr. Res. (in press).

Davidson, J.R.T. (1990) Continuation treatment of panic disorder with high-potency benzodiazepines. J. Clin. Psychiatry 15, 31-37.

Fabre, L.F. and McLendon, D.M. (1980) A double-blind study comparing the efficacy and safety of alprazolam with imipramine and placebo in primary depression. Curr. Ther. Res. 27, 474482.

Feighner, J.P., Aden, G.C., Fabre, L.F., Rickels, K. and Smith, W.T. (1983) Comparison of alprazolam, imipramine and placebo in the treatment of depression. J. Am. Med. Assoc. $249,3057-3064$.

Gloger, S., Grunhaus, L., Birmacher, B. and Troudart, T. (1981) Treatment of spontaneous panic attacks with clomipramine. Am. J. Psychiatry I38, 1215-1217.

Gorman, J.M., Liebowitz, M.R., Fyer, A.J., Goetz, D., Campeas, R.B., Fyer, M.R., Davies, S.O. and Klein, D.F. (1987) An open trial of fluoxetine in the treatment of panic attacks. J. Clin. Psychopharmacol. 7, 329-332.

Greenblatt, D.J. and Shader, R.I. (1974) Benzodiazepines in Clinical Practice. Raven Press, New York, NY.

Grunhaus, L., Gloger, S. and Birmacher, B. (1984) Clomipramine treatment for panic attacks in patients with mitral valve prolapse. J. Clin. Psychiatry 45, 25-27.

Klein, D.F. and Fink, M. (1962) Psychiatric reaction patterns to imipramine. Am. J. Psychiatry 119, 432-438.
Klerman, G.L., Coleman, J.H. and Purpura, R.P. (1986) The design and conduct of the Upjohn Cross-National Collaborative Panic Study. Psychopharmacol. Bull. 22, 59-64.

Kolin, I.S. and Linet, O.I. (1981) Double blind comparison of alprazolam and diazepam for subchronic withdrawal from alcohol. J. Clin. Psychiatry 42, 169-173.

Levine, J. and Schooler, N.R. (1986) SAFTEE: A technique for the systematic assessment of side effects in clinical trials. Psychopharmacol. Bull. 22, 343-381.

Lydiard, R.B., Laraia, M.T., Ballenger, J.C. and Howell, E.F. (1987) Emergence of depressive symptoms in patients receiving alprazolam for panic disorder. Am. J. Psychiatry 144, 664-665.

Mavissakalian, M. and Michelson, L. (1986) Agoraphobia: relative and combined effectivness of therapist in vivo exposure and imipramine. J. Clin. Psychiatry 47, 117-122.

Mendels, J. and Schless, A.P. (1986) Comparative efficacy of alprazolam, imipramine and placebo administered once a day in treating depressed patients. J. Clin. Psychiatry 47 , $357-361$.

Noyes, R. Jr., Dupont, R.L. Jr., Pecknold, J.C., Rifkin, A., Rubin, R.T., Swinson, R.P., Ballenger, J.C. and Burrows, G.D. (1988) Alprazolam in panic disorder and agoraphobia: Results from a multicenter trial II. Patient acceptance, side effects and safety. Arch. Gen. Psychiatry 45, 423-428.

Noyes, R., Garvey, M.J., Cook, B. and Samuelson, L. (1989) Problems with tricyclic antidepressant use in patients with panic disorder or agoraphobia: results of a naturalistic follow-up study. J. Clin. Psychiatry 50, 163-169.

Pecknold, J.C., Swinson, R.P., Kuch, K. and Lewis, C.P. (1988) Alprazolam in panic disorder and agoraphobia: Results from a multicenter trial III. Discontinuation effects. Arch. Gen. Psychiatry 45, 429436.

Pollack, M.H. (1990) Long-term management of panic disorder. J. Clin. Psychiatry 51, 11-13.

Rizley, R., Kahn, R.J., McNair, D.M. and Frankenthaler, L.M. (1986) A comparison of alprazolam and imipramine in the treatment of agoraphobia and panic disorder. Psychopharmacol. Bull. 2, 167-172.

Schweizer, E., Rickels, K., Weiss, S. and Zavodnick, S. (1993) Maintenance drug treatment of panic disorder I. Results of a prospective, placebo-controlled comparison of alprazolam and imipramine. Arch. Gen. Psychiatry 50, 51-60.

Sheehan, D.V., Ballenger, J.C. and Jacobsen, G. (1980) Treatment of endogenous anxiety with phobic, hysterical and hypochondriacal symptoms. Arch. Gen. Psychiatry 37, $51-59$.

Sheehan, D.V., Claycomb, J.B. and Surman, O.S. (1984) The relative efficacy of alprazolam, phenelzine and imipramine in treating panic attacks and phobias. 137th Annual Meeting of the American Psychiatric Association, Los Angeles, CA.

Strahan, A., Rosenthal, J., Kaswan, M. and Winston, A. (1985) Three case reports of acute paroxysmal excitement associated with alprazolam treatment. Am. J. Psychiatry 142, 859-861.

Tesar, G.E. (1990) High potency benzodiazepines for shortterm management of panic disorder: the U.S. experience. J. Clin. Psychiatry 51, 4-10. 